\title{
Impact of Maternal Lateral Tilt On Cardiac Output During Caesarean Section Under Spinal Anaesthesia: A Prospective Observational Study
}

Chiara Sonnino ( $\nabla$ chiara.sonnino@policlinicogemelli.it )

IRCCS Fondazione Policlinico Universitario A. Gemelli

Luciano Frassanito

IRCCS Fondazione Policlinico Universitario A. Gemelli

Alessandra Piersanti

IRCCS Fondazione Policlinico Universitario A. Gemelli

Pietro Paolo Giuri

IRCCS Fondazione Policlinico Universitario A. Gemelli

Bruno Antonio Zanfini

IRCCS Fondazione Policlinico Universitario A. Gemelli

Stefano Catarci

IRCCS Fondazione Policlinico Universitario A. Gemelli

Gaetano Draisci

IRCCS Fondazione Policlinico Universitario A. Gemelli

\section{Research Article}

Keywords: Left uterine displacement, cardiac output, noninvasive hemodynamic monitoring, cesarean delivery, spinal anesthesia

Posted Date: January 3rd, 2022

DOI: https://doi.org/10.21203/rs.3.rs-1149869/v1

License: (a) (1) This work is licensed under a Creative Commons Attribution 4.0 International License. Read Full License

Version of Record: A version of this preprint was published at BMC Anesthesiology on April 11th, 2022. See the published version at https://doi.org/10.1186/s12871-022-01640-6. 


\section{Abstract}

Background: Left uterine displacement (LUD) has been questioned as an effective strategy to prevent aortocaval compression after spinal anesthesia (SA) for cesarean delivery (CD). We tested if LUD has a significant impact on cardiac output (CO) in patients undergoing $C D$ under SA during continuous noninvasive hemodynamic monitoring.

Methods: Forty-six patients were included in the final analysis. We considered 4 timepoints of 5 minutes each: T1=baseline with LUD; T2=baseline without LUD; T3=after SA with LUD; T4=after SA without LUD. LUD was then repositioned for CD. Primary outcome was to test if CO decreased from T3 to T4. We also compared CO between T1 and T2 and other hemodynamic variables: mean, systolic and diastolic blood pressure (respectively MAP, SAP and DAP), heart rate (HR), stroke volume (SV), stroke volume variation $(\mathrm{SVV})$, pulse pressure variation (PPV), contractility (dP/dt), dynamic arterial elastance $\left(\mathrm{Ea}_{\mathrm{dyn}}\right)$ at the different timepoints. Data on fetal Apgar scores and umbilical arterial and venous $\mathrm{pH}$ were collected.

Results: CO did not vary from T3 to T4 [CO mean difference $-0.02 \mathrm{~L} / \mathrm{min}[95 \% \mathrm{Cl}-0.88$ to $0.82 ; \mathrm{p}=1]$. No significant variation was registered for any variable at any timepoint.

Conclusions: LUD did not show a significant impact on CO during continuous hemodynamic monitoring after SA for CD.

Trial registration: (retrospectively registered on 03/12/2021) NCT05143684.

\section{Introduction}

Since 1953, the gravid uterus in pregnancies at term has been recognized as a cause of aortic and caval compression in the supine position. ${ }^{1}$ Later, experiments with venograms provided a visual evidence of the impaired venous return suggesting the adoption of the left tilt uterine tilt in clinical practice. ${ }^{2}$ In most patients, venoconstriction of the lower limbs allows complete compensation, ${ }^{3}$ but sympathetic blockade following spinal anesthesia (SA) for cesarean delivery (CD) blunts cardiovascular compensatory mechanisms, exacerbating maternal hypotension and neonatal depression. ${ }^{4,5}$

The introduction of a $15^{\circ}$ left uterine displacement (LUD) was proposed for the first time by Crawford and colleagues in 1972, as a result of their experiments on 150 women undergoing CD under general anesthesia. ${ }^{6}$ However, there is no consensus on whether tilting the table improves maternal or neonatal outcome. In fact, not only LUD is rarely effectively achieved in every day practice ${ }^{7,8}$, making its efficacy in preventing aortocaval compression unreliable, but it may make the operation more difficult for the surgeon.

The introduction of optimized vasopressor and fluid therapy posed questions on its effective utility. ${ }^{9}$

A Cochrane review found no differences in hypotensive events between supine and LUD patients. ${ }^{10}$ 
Lee and colleagues measured CO, stroke volume (SV) and systemic vascular resistances by suprasternal Doppler ultrasound in not anesthetized parturients with four levels of left lateral tilt $\left(0^{\circ}, 7.5^{\circ}, 15^{\circ}\right.$ and $\left.90^{\circ}\right),{ }^{11}$ showing that aortocaval compression can be effectively minimized by the use of a left lateral tilt of $15^{\circ}$ or greater.

On the other hand, Tsai and colleagues showed that NICOM hemodynamic monitoring could not detect any difference in cardiac index between patients with left-tilt and supine ${ }^{12}$ while Chungsamarnyart showed only modest hemodynamic advantages (higher $\mathrm{CO}$, less hypotension, higher $\mathrm{dP} / \mathrm{dT}$ ) with predelivery LUD. ${ }^{13}$

The aim of this study was to evaluate if CO varies significantly removing LUD after SA for CD during continuous non-invasive monitoring. We also compared values of mean arterial pressure (MAP), systolic arterial pressure (SAP), diastolic arterial pressure (DAP), SV, stroke volume variation (SVV), heart rate $(\mathrm{HR})$, pulse pressure variation $(\mathrm{PPV})$, contractility $\left(\mathrm{dP} / \mathrm{dt}_{\mathrm{max}}\right)$ and dynamic arterial elastance $\left(\mathrm{Ea}_{\mathrm{dyn}}=\right.$ PPV/SVV) with and without LUD before and after SA to asses for significative differences.

\section{Matherials And Methods}

This is a prospective observational study testing as primary outcome if $\mathrm{CO}$ decreases significantly after LUD removal in patients under SA for CD.

The trial was retrospectively registered on ClinicalTrials.gov with protocol number NCT05143684 on $03 / 12 / 2021$.

The Internal Ethic Committee approved the study (ID 3197, protocol N 27861/20).

Data were gathered from pregnant patients who, in addition to standard monitoring (5-lead electrocardiogram, pulse oximetry, non-invasive intermittent blood pressure, urine output), underwent perioperative non-invasive monitoring by ClearSight system on the Edwards Lifesciences HemoSphere platform (Edwards Lifesciences, Irvine, CA) at delivery suite of IRCCS Policlinico Agostino Gemelli Foundation of Rome, Italy from June 1st 2020 to July 31st 2020. The ClearSight system consists of a finger cuff positioned at the middle phalanx of the third finger of the non-dominant hand of the patient, that allows for advanced hemodynamic parameters and continuous noninvasive blood pressure. ${ }^{14}$

The parameters we evaluated from the ClearSight system for the analysis were CO, MAP, SAP, DAP, SV, $\mathrm{SVV}, \mathrm{HR}, \mathrm{PPV}, \mathrm{dP} / \mathrm{dt}_{\max }$ and PPV/SVV recorded at 20 seconds-intervals.

Inclusion criteria consisted in pregnant patients at term (36th to 40th week of gestation) scheduled for elective CD under SA.

Exclusion criteria were: age $<18$ years, American Society of Anesthesiologists score $>3$, cardiac arrhythmias or aortic regurgitation, pregnancy-induced hypertension, pre-eclampsia, body mass index 
$(\mathrm{BMI})>35 \mathrm{~kg} / \mathrm{m}^{2}$, foetal complications, coagulation disorders or contraindication to neuraxial block, emergency surgery, preoperative infection, patient's refusal.

We considered 4 timepoints. We indicated as T1 the baseline values recorded for 5 minutes, after initial stabilization of parameters, with the patient laying down on the operating table with LUD. At T2, LUD was removed and we considered for the analysis hemodynamic data of the subsequent 5 minutes. We indicated as T3 the 5 minutes following SA with a satisfactory sensory block and as T4 the subsequent 5 minutes following LUD removal. Figure 1 summarizes the timepoints of our analysis. LUD was accomplished by positioning a wooden wedge wrapped with cotton, to make it comfortable, and medical sheets with a measured angle of $15^{\circ}$ under the right flank of the laying down patient. In all patients, after T4 the $15^{\circ}$ wooden wedge was positioned again and surgery was performed with LUD.

Anesthesia was delivered in sitting position using a 25-G Whitacre spinal needle, at the L3-4 vertebral interspace, with hyperbaric $0.5 \%$ bupivacaine, sufentanil $5 \mathrm{mcg}$ and morphine $100 \mathrm{mcg}$. The bupivacaine dose administered was standardized according to patient's height, as usual practice in our Institution: 8 $\mathrm{mg}$ for women $<160 \mathrm{~cm}$ tall, $9 \mathrm{mg}$ for women between 160 and $170 \mathrm{~cm}$, and $10 \mathrm{mg}$ for those $>170 \mathrm{~cm}$. Once the anesthetic procedure was completed, all patients received a rapid crystalloid co-load of $7 \mathrm{ml} / \mathrm{kg}$ over 10 minutes. During surgery and after delivery, fluid management was left to the attending anesthesiologist.

The attending anesthesiologist was blinded to the advanced hemodynamic parameters from the ClearSight system except for the continuous BP values. We defined hypotension as an absolute value of MAP $<65 \mathrm{mmHg}$. This value was considered as trigger for the attending anesthesiologist for the administration of norepinephrine $5 \mathrm{mcg}$. Norepinephrine boluses were repeated to reach a MAP $>65 \mathrm{mmHg}$. Bradycardia was defined as a heart rate of $<60 \mathrm{bpm}$. Atropine $0.5 \mathrm{mg}$ was administered for the treatment of bradycardia combined with hypotension, or for an absolute value of heart rate $<45 \mathrm{bpm}$. After delivery, Oxytocin was administered to facilitate the uterine contraction.

We also evaluated the impact of maternal blood pressure and $\mathrm{CO}$ on fetal outcome collecting neonatal Apgar scores at 1 and 5 minutes after birth, and umbilical cord arterial and venous $\mathrm{pH}$.

\section{Statistical analysis}

Our primary outcome was to verify if LUD had a significant impact on $\mathrm{CO}$ from $\mathrm{T} 3$ to $\mathrm{T} 4$, as recorded by the non-invasive continuous hemodynamic monitoring system.

Secondary outcomes included the mean differences in hemodynamic variables (SAP, DAP, SV, SVV, HR, $\mathrm{PPV}, \mathrm{dP} / \mathrm{dt}_{\max }$ and PPV/SVV $\left(\mathrm{Ea}_{\mathrm{dyn}}\right)$ at baseline and after SA.

Repeated measure analysis of variance (ANOVA) or its non-parametric alternative Friedman test for nonnormally distributed variables were used to compare patients' longitudinal hemodynamic data from baseline until ten minutes after SA was performed. Sphericity assumption was not violated and it was 
assessed with the Mauchly's test $(p>0.05)$. Bonferroni's adjustments for multiple comparisons was applied for pairwise comparisons among group means.

Normally distributed continuous variables were reported as mean ( \pm standard deviation, SD), and as median and interquartile range (IQR) those not normally distributed. Categorical variables were reported as number and percentage. Shapiro-Wilk test was used to assess normality of data distribution and the equality of variances was verified with the variance ratio test. Statistical significance level was $0.05 \%$.

We estimated the sample size based on the $C O$ reported in a recently published randomized controlled trial comparing patients with and without LUD during CD under SA. ${ }^{13}$ The reported mean CO $7.20 \pm 1.78$ $\mathrm{L} / \mathrm{min}$ in patients with LUD and $6.23 \pm 1.44 \mathrm{~L} / \mathrm{min}$ in patients without LUD.

Considering a significance level of 0.05 and a power of the test of 0.90 (https://clincalc.com/stats/samplesize.aspx), we estimated a minimum sample size of 35 patients to detect the same variation of $\mathrm{CO}$ after LUD removal. We decided to include all patients whose data were recorded and complete, who did not meet any exclusion criteria, for a total of 46 patients, to account for dropouts.

\section{Results}

We screened 90 pregnant women at term scheduled for elective CD. Forty cases were excluded because they did not meet the inclusion criteria (not at term $\mathrm{N}=23$, not elective procedures $\mathrm{N}=17$ ). Four patients were excluded due to incomplete records of the data. We used for the final analysis data from 46 women, including 6 twin pregnancies with mean gestational age of $38+/-2$ weeks. The consort diagram is represented in Figure 2. All the included patients had successful sensory block to T4 that allowed surgery to be completed.

Demographic and intraoperative data, together with fetal Apgar scores and umbilical venous (UV) and arterial (UA) blood gas analysis are summarized in Table 1. 
Table 1

Demographic and intraoperative data of all patients. Data are expressed as $\mathrm{n}(\%)$, mean \pm SD or median (range). LUD: left uterine displacement

\begin{tabular}{|ll|}
\hline & $\mathbf{n}=46$ \\
\hline Age (year) & $36( \pm 6)$ \\
\hline Height $(\mathrm{m})$ & $1.63( \pm 0.1)$ \\
\hline Weight $(\mathrm{Kg})$ & $74( \pm 12)$ \\
\hline Body Mass Index $(\mathrm{Kg} / \mathrm{m} 2)$ & $27(24-30)$ \\
\hline Twin pregnancy & $6(13 \%)$ \\
\hline $0.5 \%$ hyperbaric bupivacaine dose $(\mathrm{mg})$ & $9( \pm 1)$ \\
\hline Crystalloid co-load after neuraxial anesthesia $(\mathrm{mL})$ & $516( \pm 83)$ \\
\hline Norepinephrine dose after neuraxial anesthesia with LUD (mcg) & $13( \pm 6)(\mathrm{n}=22)$ \\
\hline Norepinephrine dose after neuraxial anesthesia without LUD & $9( \pm 6)(\mathrm{n}=20)$ \\
\hline Total norepinephrine dose during surgery (mcg) & $35(15-50)(\mathrm{n}=38)$ \\
\hline Operative time (min) & $84( \pm 16)$ \\
\hline Apgar 1 minute & $8.6(2-9)$ \\
\hline Apgar 5 minutes & $9.4(2-10)$ \\
\hline UVpH & $7.32( \pm 0.05)$ \\
\hline UApH & $7.29( \pm 0.09)$ \\
\hline
\end{tabular}

We did not find any significant CO variation after LUD removal after SA, nor after LUD removal at baseline [CO mean difference 0.34 (SE 0.32) L/min [95\% Cl -0.05; 1.19] at baseline vs. -0.028 (SE 0.32) L/min [95\% $\mathrm{Cl}-0.08 ; 0.82] ; p=1.00]$ after SA (Figure 3).

We did not find significant mean differences with LUD and after its removal at baseline or after SA for any of the hemodynamic variables considered ( $p>0.20)$ (Figure 4), except for SAP from T1 to T2 [SAP mean difference 9.78 (SE 3.16) $\mathrm{mmHg}[95 \% \mathrm{Cl} 1.33 ; 18.23 ; p=0.014]$.

All the hemodynamic values at different timepoints are reported in Table 2. 
Table 2

- Hemodynamic variables at the different timepoints. Data are expressed as mean \pm SD or median (range). Cardiac Contractility assessed as $\mathrm{dP} / \mathrm{dtmax}$. Dynamic Arterial Elastance (Eadyn) assessed as PPV/SVV. LUD: left uterine displacement; SA: spinal anesthesia; UVpH: umbilical vein $\mathrm{pH}$; UApH: umbilical artery $\mathrm{pH}$. T1=baseline with LUD; T2=baseline without LUD; T3=after SA with LUD; T4=after SA without LUD.

\begin{tabular}{|lllll|}
\hline & T1 & T2 & T3 & T4 \\
\hline CO (L/min) & $5,3( \pm 1,5)$ & $5,6( \pm 1,4)$ & $5,4(4,3-6,4)$ & $5,4(4,2-6,5)$ \\
\hline MAP (mmHg) & $93( \pm 8)$ & $98( \pm 8)$ & $82(75-89)$ & $80(71-86)$ \\
\hline SAP (mmHg) & $120( \pm 13)$ & $127( \pm 12)$ & $108(99-118)$ & $105(95,-113)$ \\
\hline DAP (mmHg) & $77( \pm 7)$ & $80( \pm 7)$ & $68(64-74)$ & $66(60-71)$ \\
\hline HR (bpm) & $84( \pm 11)$ & $87( \pm 11)$ & $88( \pm 14)$ & $83( \pm 14)$ \\
\hline SV (ml/b) & $63( \pm 16)$ & $65( \pm 15)$ & $64(52-72)$ & $66(52-76)$ \\
SWV (\%) & $12(10-14)$ & $12(11-14,64)$ & $13(11-14)$ & $10(9-12)$ \\
\hline PPV (\%) & $12(9-15)$ & $14(13-17)$ & $16( \pm 4)$ & $14( \pm 4)$ \\
\hline dP/dt (mmHg/sec) & $536(426-621)$ & $566(501-671)$ & $466(383-603)$ & $443(36-528)$ \\
\hline Eadyn & $1(0,9-1,2)$ & $1,1(1,0-1,2)$ & $12(1,1-1,4)$ & $1,2(1,-1,5)$ \\
\hline
\end{tabular}

At T3, during the first 5 minutes after SA, 22 patients received norepinephrine to treat hypotension and at T4, during the subsequent 5 minutes after removal of LUD, vasopressor was administered to 20 patients [mean dose 13 ( $\pm 5.87 \mathrm{SD}) \mathrm{mcg}$ at T3 vs. $9(5.91) \mathrm{mcg}$ at T4; $\mathrm{p}=0.06[95 \% \mathrm{Cl}(-0.26+8.75)] .14$ patients (30\%) did not need vasopressors during the first 10 minutes after SA.

Mean Apgar score at 1 and 5 minutes were, respectively, $8.6(\min 2 ; \max 9)$ and $9.4(\min 2 ; \max 10)$, with one case of Apgar score of 2 at 1 and 5 minutes in a baby with trisomy 18 disease born at 36 weeks of gestation. Mean UV pH was 7.32 ( \pm 0.05$)$, and mean UA pH was $7.29( \pm 0.09)$

\section{Discussion}

In this prospective observational study, we found that under continuous hemodynamic monitoring, $\mathrm{CO}$ does not show any significant variation after LUD removal under SA for CD. LUD showed no impact on $\mathrm{CO}$ neither at baseline, before SA. Blood pressure, HR, SV, SVV, PPV, $\mathrm{dP} / \mathrm{dt}_{\max }$ and $\mathrm{Ea}_{\mathrm{dyn}}$ did not vary significantly with and without LUD either at baseline or after SA.

Of 46 patients, 22 (48\%) needed vasopressor support right after SA with LUD, and 20 (43\%) needed vasopressor support after LUD removal under SA. The total amount of norepinephrine was significantly higher after LUD removal under SA, but SAP, MAP and DAP were not significantly influenced. This may suggest that, even if LUD may have a role in maintaining MAP, prompt vasopressor administration is able to correct hypotensive events even without LUD. We should consider that in everyday practice blood 
pressure during $C D$ is not measured continuously, but international recommendations suggest noninvasive blood pressure measurements every minute and prophylactic vasopressor infusion ${ }^{15}$. In 2017, Lee and co-authors showed that optimal fluid and vasopressor therapy controlled the component of hypotension due to the aortocaval compression by the gravid uterus without consequences for the foetus $^{16}$.

Previous studies showed mixed results on the hemodynamic impact of LUD. ${ }^{8-20}$ A Cochrane review showed that LUD does not have any impact on non-invasively measured blood pressure. ${ }^{10}$ Some authors with hemodynamic monitoring showed that LUD determines minimal improvement in term pregnancy without anesthesia, ${ }^{17,18}$ while Lee and colleagues reported a better hemodynamic profile with LUD. ${ }^{11}$ In women undergoing SA for CD, LUD showed to improve $C O$ and the overall hemodynamic equilibrium. ${ }^{18}$

Recently, Chungsamarnyart and colleagues published their randomized-controlled trial comparing noninvasive monitoring of $\mathrm{CO}$ in patients with LUD and without LUD, showing that LUD provides modest hemodynamic advantages (higher $\mathrm{CO}$, less hypotension, higher $\mathrm{dP} / \mathrm{dT}$ ) pre-delivery. The results support maternal hemodynamic benefits of LUD until delivery in women with term pregnancies undergoing $C D$ with $S A B^{12}$. Preventing hypotension and hemodynamic derangement after $S A$ for $C D$ is a challenge for the obstetric anesthesiologist in order to avoid maternal and fetal complications.

In this study we open new questions on the hemodynamic benefit of LUD.

This study has some limitations. Firstly, its design does not include a control group, but patients act as their own control after LUD removal before and after SA.

Also, we defined hypotensive events as MAP $<65 \mathrm{mmHg}$, even if in obstetric anesthesia the most common definition of hypotension refers to SAP $(<80 \%$ baseline or $<100 \mathrm{mmHg}) .{ }^{21,22}$ Nevertheless, the role of MAP as determinant of organ perfusion is well known. ${ }^{22,23}$

\section{Conclusions}

CO does not decrease significantly after LUD removal in patients under SA for CD during continuous hemodynamic monitoring with HPI. Optimization of fluid and vasopressor therapy may be sufficient to prevent aorto-caval compression by the gravid uterus and the consequent reduction of venous return after SA for CD.

\section{List Of Abbeviations}

LUD: Left uterine displacement

SA: spinal anesthesia

CD: cesarean delivery 
CO: cardiac output

MAP: mean arterial pressure

SAP: systolic arterial pressure

DAP: diastolic arterial pressure

HR: heart rate

SV: stroke volume

SVV: stroke volume variation

PPV: pulse pressure variation

$\mathrm{dP} / \mathrm{dt}$ : contractility

$\mathrm{Ea}_{\mathrm{dyn}}:$ dynamic arterial elastance

SD: standard deviation

IQR: interquartile range

UV: umbilical venous

UA: umbilical arterial

\section{Declarations}

Ethics approval and consent to participate: The Internal Ethic Committee (Comitato Etico Fondazione Policlinico Universitario Agostino Gemelli IRCCS - Università Cattolica del Sacro Cuore) approved the study (ID 3197, protocol N 27861/20), all patients expressed written informed consent.

This protocol has been conducted in accordance to Good Clinical Practice guidelines and to the princicples of the Helsinki Declaration and to the current legislation.

Consent for publication: Not applicable

Availability of data and materials: The datasets used and/or analysed during the current study are available from the corresponding author on reasonable request.

Competing interests: Dr Sonnino, Piersanti, Giuri, Zanfini, Catarci and Professor Draisci have no competing interests to declare.

Dr Frassanito received an honorarium from Edwards Lifesciences Ltd for scientific advice. 
Funding: Not applicable

Authors' contributions:

CS designed the study, recruited patients, collected the data, drafted the manuscript and reviewed the final manuscript.

LF designed the study, recruited patients, collected the data, and reviewed the final manuscript.

AP helped design the study, analysed data, and reviewed the final manuscript.

PPG helped design the study, recruit patients, collect the data, and review the final manuscript.

BAZ helped design the study, recruit patients, collect the data, and review the final manuscript.

SC helped design the study, recruit patients, collect the data, and review the final manuscript.

Author name: Pietro Paolo Giuri, MD. This author helped design the study, recruit patients, collect the data, and review the final manuscript.

GD helped design the study, recruit the patients, collect data, and review the final manuscript.

Acknowledgements: Not applicable

Authors' information: CS, LF, PPG, BAZ, and SC are registered anaesthesiologists in the Unit of Obstetric and Gynecologic Anesthesia, IRCCS Fondazione Policlinico Universitario Agostino Gemelli. GD is the head of the Unit of Obstetric and Gynecologic Anesthesia, IRCCS Fondazione Policlinico Universitario Agostino Gemelli. AP is a resident in anesthesia and Intensive Care at IRCCS Fondazione Policlinico Universitario Agostino Gemelli with advanced formation in statistical analysis at Università Cattolica del Sacro Cuore of Rome and at Istituto Mario Negri.

\section{References}

1. Howard BK, Goodson JH, Mengert WF: Supine hypotensive syndrome in late pregnancy. Obstet Gynecol 1953; 1:371-7]. Asmussen E, Christensen EH, Neilsen M. Regulation of circulation in different postures. Surgery 1940;8:604-7.

2. Scott DB. Inferior vena caval occlusion in late pregnancy and its importance in anaesthesia. $\mathrm{Br} \mathrm{J}$ Anaesth 1968; 40:120-128.

3. Kinsella SM, Lohmann G: Supine hypotensive syndrome. Obstet Gynecol 1994; 83:774-88 Lee AJ, Landau R. Aortocaval Compression Syndrome: Time to Revisit Certain Dogmas. Anesthesia \& Analgesia. 2017 Dec; 125(6):1975-85.

4. Holmes F: The supine hypotensive syndrome: 1960. Anaesthesia 1995; 50:972-7. 
5. Goodlin RC: Aortocaval compression during cesarean section: A cause of newborn depression. Obstet Gynecol 1971; 37:702-5.

6. Crawford JS, Burton M, Davies P: Time and lateral tilt at Caesarean section. Br J Anaesth 1972; 44:477-84.

7. Jones SJ, Kinsella SM, Donald FA: Comparison of measured and estimated angles of table tilt at Caesarean section. Br J Anaesth 2003; 90:86-7.

8. Aust $\mathrm{H}$, Koehler $\mathrm{S}$, Kuehnert $\mathrm{M}$, Wiesmann $\mathrm{T}$ : Guideline- recommended $15^{\circ}$ left lateral table tilt during cesarean section in regional anesthesia-practical aspects: An observational study. J Clin Anesth 2016; 32:47-53.

9. Left Lateral Table Tilt for Elective Cesarean Delivery under Spinal Anesthesia Has No Effect on Neonatal Acid-Base Status.

10. Cluver C, Novikova N, Hofmeyr GJ, Hall DR. Maternal position during caesarean section for preventing maternal and neonatal complications. Cochrane Database Syst Rev 2013:CD 007623.

11. Lee SW, Khaw KS, Ngan Kee WD, Leung TY, Critchley LA. Haemodynamic effects from aortocaval compression at different angles of lateral tilt in non-labouring term pregnant women. $\mathrm{Br} \mathrm{J}$ Anaesth 2012;109:950-6.

12. Tsai S-E, Yeh PH, Hsu PK, et al. Continuous haemodynamic effects of left tilting and supine positions during Caesarean section under spinal anaesthesia with a noninvasive cardiac output monitor system. Eur J Anaesthesiol 2019; 36:72-74.

13. Chungsamarnyart $Y$, Wacharasint $P$, Carvalho $B$. Hemodynamic profiles with and without left uterine displacement: A T randomized study in term pregnancies receiving subarachnoid blockade for cesarean delivery. J Clin Anesth. 2020;64:109796.

14. Kim SH, Lilot M, Sidhu KS, Rinehart J, Yu Z, Canales C, Cannesson M. Accuracy and precision of continuous noninvasive arterial pressure monitoring compared with invasive arterial pressure: a systematic review and meta-analysis. Anesthesiology. 2014;120:1080-97.

15. Kinsella SM, Carvalho B, Dyer RA, et al. Consensus Statement Collaborators. International consensus statement on the management of hypotension with vasopressors during caesarean section under spinal anaesthesia. Anaesthesia. 2018;73(1):71-92.

16. Lee AJ, Landau R, Mattingly JL. Left Lateral Table Tilt for Elective Cesarean Delivery under Spinal Anesthesia Has No Effect on Neonatal Acid-Base Status. Anesthesiology. 2017;127:241-9.

17. Practice guidelines for obstetric anesthesia: an updated report by the American Society of Anesthesiologists Task Force on obstetric anesthesia and the Society for Obstetric Anesthesia and Perinatology. Anesthesiology. 2016;124:270-300.

18. Bamber JH, Dresner M. Aortocaval compression in pregnancy: the effect of changing the degree and direction of lateral tilt on maternal cardiac output. Anesth Analg 2003;97:256-8.

19. Higuchi H, Takagi S, Zhang K, Furui I, Ozaki M. Effect of lateral tilt angle on the volume of the abdominal aorta and inferior vena cava in pregnant and nonpregnant women determined by magnetic resonance imaging. Anesthesiology 2015;122:286-93. 
20. Hasanin A, Soryal R, Kaddah T, et al. Hemodynamic effects of lateral tilt before and after spinal anesthesia during cesarean delivery: an observational study. BMC Anesthesiol 2018;18:8.

21. Klohr S, Roth R, Hofmann T, Rossaint R, Heesen M. Definitions of hypotension after spinal anaesthesia for caesarean section: literature search and application to parturients. Acta Anaesthesiologica Scandinavica 2010; 54: 909-21.

22. Ackland GL, Brudney CS, Cecconi M, et al. Perioperative Quality Initiative-3 workgroup; POQI chairs; Physiology group; Preoperative blood pressure group; Intraoperative blood pressure group; Postoperative blood pressure group. Perioperative Quality Initiative consensus statement on the physiology of arterial blood pressure control in perioperative medicine. $\mathrm{Br} \mathrm{J}$ Anaesth. 2019 May;122(5):542-551.

\section{Figures}

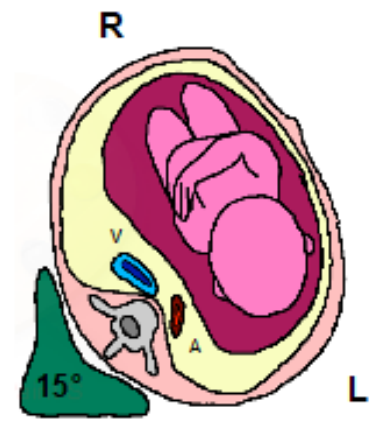

T1

Basal monitoring with LUD
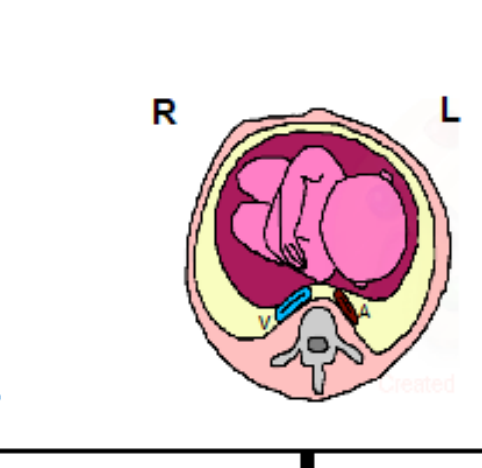

T2

Basal monitoring without LUD

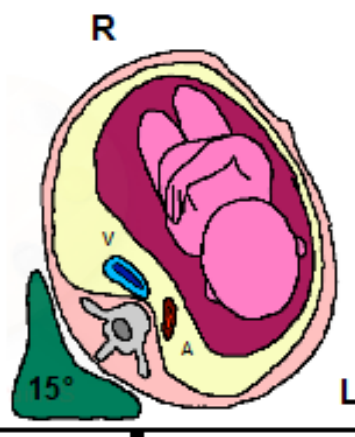

T3

Monitoring after SA

with LUD
$\mathbf{R}$

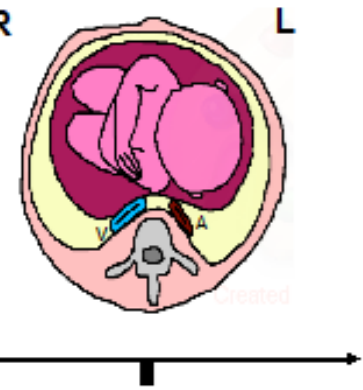

T4

Monitoring after SA without LUD

\section{Figure 1}

Description of timepoints for comparison of hemodynamic variables. V: vena cava; A: aorta; LUD: left uterine displacement; SA: spinal anesthesia. $\mathrm{R}=$ right; $\mathrm{L}=$ left. 


\section{CONSORT 2010 Flow Diagram}

\section{Enrollment}

Assessed for eligibility ( $n=90$ )

uded $(n=40)$

- Not elective procedures $(n=17)$

- Not at term pregnancies $(n=23)$

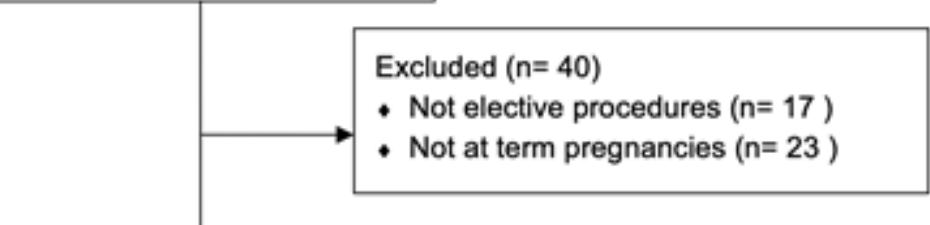

Enrolled $(n=50)$
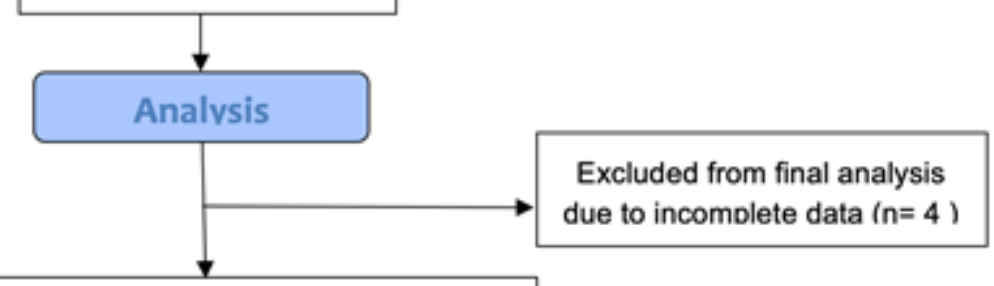

Included in the final analysis $n=46$

\section{Figure 2}

Consort diagram.

\section{CO}

9

7

3

1

T1

T2

T3

T4 


\section{Figure 3}

Mean values of $\mathrm{CO}$ at the single timepoints in $\mathrm{ml} / \mathrm{min} . \mathrm{CO}=$ cardiac output. $\mathrm{T} 1=$ baseline with left uterine displacement (LUD); T2= baseline without LUD; T3= after spinal anesthesia (SA) with LUD; T4= after SA without LUD.
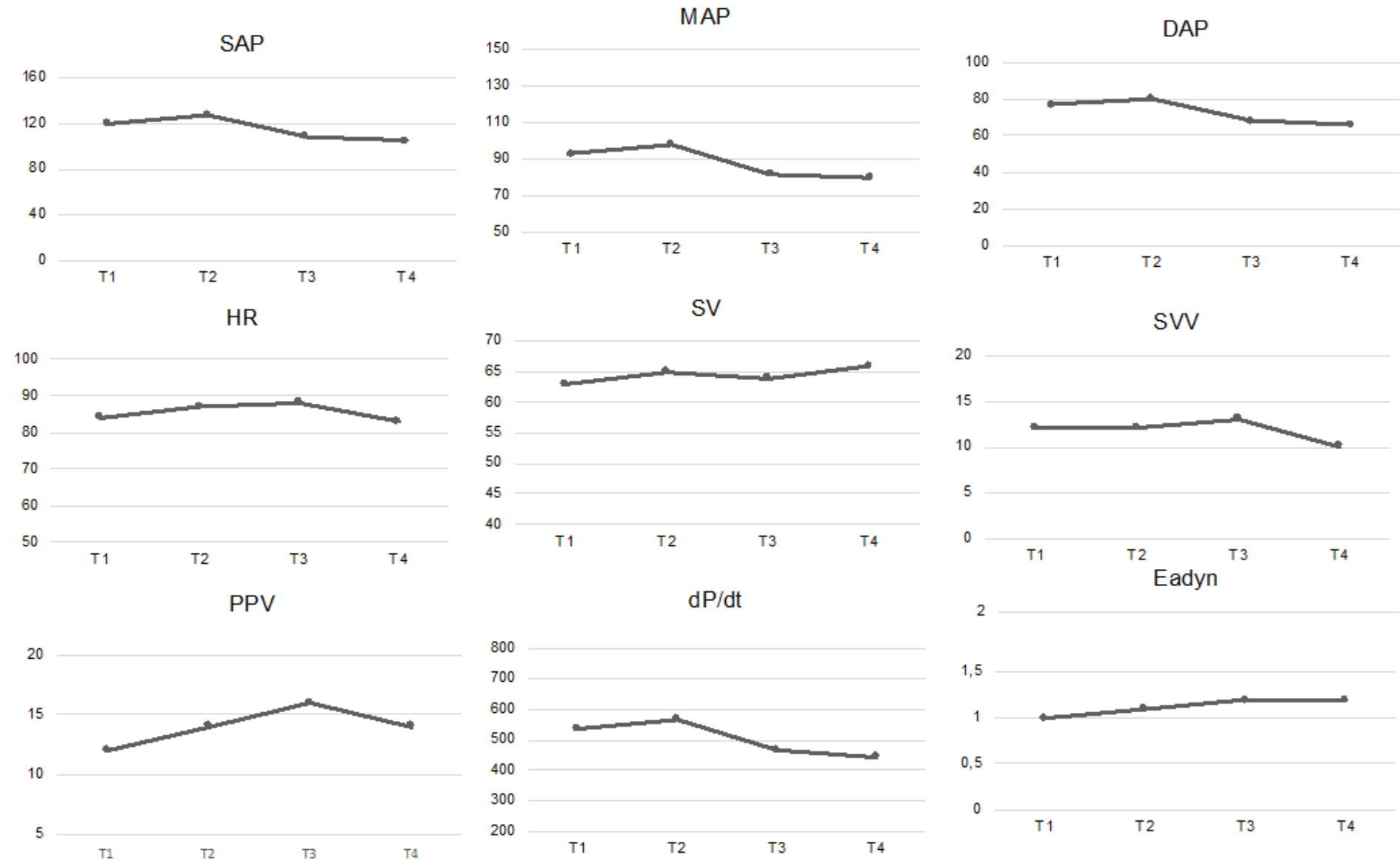

\section{Figure 4}

Mean values of main hemodynamic variables at the single timepoints: $\mathrm{T} 1=$ baseline with left uterine displacement (LUD); T2= baseline without LUD; T3= after spinal anesthesia (SA) with LUD; T4= after SA without LUD. SAP= systolic arterial pressure in $\mathrm{mmHg}$; MAP= mean arterial pressure in $\mathrm{mmHg}$; $\mathrm{DAP}=$ diastolic arterial pressure in $\mathrm{mmHg} ; \mathrm{HR}=$ heart rate in bpm; SV= stroke volume in $\mathrm{ml}$; SVV= stroke volume variation in \%; PPV: pulse pressure variations in \%; $\mathrm{dP} / \mathrm{dt}$ : contractility in $\mathrm{mmHg} / \mathrm{sec}$; $\mathrm{Ea}_{\mathrm{dyn}}=$ dynamic elastance. 